\title{
PFLEGE
}

\section{Entbürokratisierung nach innen und außen}

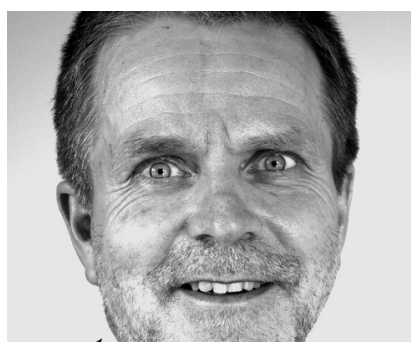

\section{VON DETLEF FRIEDRICH} Detlef Friedrich ist geschäftsführender Gesellschafter der contec $\mathrm{GmbH}$ und Geschäftsführer des Institutes für europäische Gesundheits- und Sozialwirtschaft GmbH (IEGUS). Er ist Mitinitiator und Gründungsmitglied der Deutschen Gesellschaft für Controlling in der Sozialwirtschaft (DGCS) und der Deutschen Gesellschaft für Integrierte Versorgung (DGIV).

Internet http://www.contec.de
Modellprojekte mit Sozialunternehmen, Behörden und Aufsichtsinstitutionen zeigen immer wieder, wie steinig der Weg zu einer dialogorientierten Zusammenarbeit aller ist. Angesichts knapper Ressourcen gerade in der Pflege gibt es zur Entbürokratisierung allerdings keine Alternative.

»Wir werden die Rahmenbedingungen für Pflegende und Leistungsanbieter konsequent überprüfen und entbürokratisieren, damit der eigentlichen Pflege am Menschen wieder mehr Zeit eingeräumt wird.

So steht es im Koalitionsvertrag unter dem Thema "Weiterentwicklung der Pflegeversicherung «. Ist Entbürokratisierung als gesellschaftlicher Prozess zu verstehen? Ja! Seit mehr als drei Jahrzehnten beschäftigen sich Politik, Wirtschaft und Verwaltung mit dem Thema, allerdings sind Erfolge nur in einzelnen Teilbereichen festzustellen.

Gerade in Pflegeeinrichtungen ist die Belastung durch Vorschriften, Pflichten und Regelungen keine neue Tendenz, sondern bitterer Alltag. Die Transparenzdebatte hat die Thematik der Entbürokratisierung in der Pflege wieder umfassender auf die $\mathrm{Ta}$ gesordnung gehoben. Mittlerweile ist eine Grenze erreicht, die spürbare Folgen auf die Verweildauer von Pflegenden in den Unternehmen hat. Sehr deutlich hört man immer wieder Aussagen von Pflegenden, dass sie aufgrund der umfassend zu leistenden Dokumentation aus dem Berufsfeld aussteigen. Spätestens an dieser Stelle müssen wir uns, angesichts des kommenden demografischen Wandels und sich abzeichnenden Personalmangel ernste Sorgen machen.

Das Projekt » Identifizierung von Entbürokratisierung der stationären Altenpflege in Deutschland « vom Bundesministerium für Familie, Senioren, Frauen und Jugend, zeigte erstmals umfassend erhebliche Entbürokratisierungspotenziale in der Pflegedokumentation, Haustechnik, Hauswirtschaft sowie der Personalverwaltung.

Orientiert man sich an dem Bürokratiebegriff nach Max Weber (vgl. Kasten »Die wissenschaftliche Fundierung von Bürokratie«), so soll hierdurch die Bevorzugung oder Benachteiligung Einzelner in Form von willkürlichen Entscheidungen vermie- den werden, weil sich alle an die gleichen und rational begründeten Spielregeln und Gesetze halten müssen. Eine solche Sicht ist erst einmal positiv geprägt. Doch wie viel Bürokratie ist notwendig, um die Qualität in der Pflege so zu sichern, dass die berechtigten Schutzinteressen der Verbraucher und der Mitarbeiter gewährleistet werden?

Wenn wir heute von Entbürokratisierung reden, reden wir auf der einen Seite von den Vorschriften, die von außen an Einrichtungen der Altenpflege gestellt werden und auf der anderen Seite von den bürokratischen Strukturen, die sich in den Einrichtungen selbst entwickelt haben.

Die von außen gestellten Vorschriften werden zudem durch unterschiedliche Institutionen vertreten. Sie sind nicht nur nicht widerspruchsfrei, sondern auch noch durch Interpretationsspielräume der sie vertretenden Mitarbeiterinnen und Mitarbeiter geprägt. Das eigentliche Ziel von Bürokratie, Willkür zu vermeiden, wird von Einrichtungsträgern als nicht erreicht gesehen.

\section{Was Sozialunternehmen belastet}

Schauen wir uns das Spannungsfeld an, in dem das Unternehmen steht. Der Verbraucherschutz wird von einer Reihe von Institutionen vertreten, allen voran Heimaufsicht und Medizinischem Dienst der Krankenkasse (MDK). Hinzu kommen freiwillige Initiativen, beispielsweise die Bundesinteressenvertretung der Nutzerinnen und Nutzer von Wohn- und Betreuungsangeboten im Alter und bei Behinderung (BIVA) e. V., die ihrerseits mit entsprechenden Prüfregularien eine Verbraucherorientierung im Sinne eines Gütesiegels den Einrichtungen bescheinigen. Auch dieses erfordert bürokratischen Aufwand, auch wenn er freiwillig geleistet wird. Dem Mitarbeiterschutz sind die Berufsgenossenschaften und die Arbeitsschutzbehörden primär verpflichtet, doch auch der Medizinische Dienst prüft Themen des Personalmanagements in den Einrichtungen.

Wo kommt es immer wieder zu Konflikten? Es sind nicht abgestimmte Anforderungen zwischen Heimaufsicht und MDK, aber vor allen Dingen auch Institutionen wie Feuerwehr, Bauaufsichten, Gesund- 
heitsämter, deren Anforderungen an die Einrichtungen insgesamt nicht sinnvoll aufeinander abgestimmt sind, so dass hier durch das einseitige institutionelle Verwaltungshandeln widersprüchliche Anforderungen auf die Einrichtungen zukommen. Beispiele hierfür gibt es viele.
Heimrecht vorliegt, gilt das Bundes-Heimrecht.

Ziel der in den Heimgesetzen verankerten Kooperation ist es, unter anderem die Sicherung einer angemessenen Qualität der Überwachung durch gegenseitige Information, die Koordination der Prüftätig-

\section{"Bürokratische Kreisläufe können oft nur durch selbstbewusste Einrichtungen und klare Spiel-} regeln unterbrochen werden"

Das ursprünglich im $\mathbb{2} 20$ Heimgesetz geschaffene Instrument der Arbeitsgemeinschaft verpflichtet zumindest die nach dem Heimgesetz zuständigen Behörden, die Pflegekassen, deren Landesverbände, den MDK sowie die zuständigen Träger der Sozialhilfe, eng zusammenzuarbeiten. Die im Jahr 2006 in Kraft getretene Föderalismusreform hat die Gesetzgebungszustände des Bundes, auf die Bundesländer übertragen. Diese Entscheidung wurde trotz des Protestes von vielen Fachstellen und Experten, verabschiedet. Gerade hier sind aufgrund von zukünftig 16 verschiedenen Gesetzestexten "Bürokratiebarrieren « insbesondere für bundesweit aufgestellte Anbieter zu erwarten. Solange noch kein neues Länder- keit und Maßnahmen zur Qualitätssicherung und zum Abstellen von Mängeln zu erreichen. Dieses ist zu begrüßen und hier hat es insbesondere seit der im Pflege-Weiterentwicklungsgesetz festgeschriebenen Verpflichtung des MDK zur flächendeckenden Prüfung Verbesserungen in der Kooperation mit der »Heimaufsicht « gegeben.

Die unterschiedliche Struktur der Institutionen verhindert jedoch manche Koordinationsansätze. Die anstehende Föderalismusreform leistet hier noch einmal ihren eigenen Beitrag. Viele Punkte zur Entbürokratisierung lagen als Ergebnisse des Runden Tisches vor und brauchten »nur " umgesetzt zu werden. Diese Umsetzung funktioniert nur, wenn sie in den Köpfen der Leitungen gewollt wird und in die Herzen der Sachbearbeiter Eingang findet. Doch hier tut sich Verwaltungshandeln schwer.

Gerade eine mangelnde Zusammenarbeit von Prüfinstitutionen führt in den Einrichtungen zu einem erhöhten Aufwand:

- Die erlebte Rechtsunsicherheit führt zu Unsicherheit hinsichtlich der Erwartungen bei den Prüfungen.

- Die Unsicherheit bezüglich der eigenen Kompetenzen führt dann wiederum zu erhöhtem bürokratischem Aufwand.

So entsteht ein Kreislauf, der nur durch selbstbewusste Einrichtungen und klare Spielregeln unterbrochen werden kann. Hierin unterscheiden sich die Einrichtungen, und dieses schlägt sich auf die Kosten nieder. Doch auch der schon erwähnte Effekt »Lex-Sachbearbeiter « wurde in Untersuchungen schon ermittelt.

Mit Hilfe des Standard-Kosten-Modells (SKM) - ein Konzept zur Ermittlung der Bürokratiekosten - wurden die Kosten berechnet, die durch die MDK- und Heimprüfungen in verschiedenen Einrichtungen hervorgerufen wurden. Ausgehend von dem Level der laufenden Dokumentation wurde der für den Prüfungsprozess - Vorbereitung - Begehung - Nachbereitung - einrichtungsindividuelle Aufwand der Prüfung er-

\section{Runder Tisch Pflege über Entbürokratisierungspotenziale}

Die Bundesministerin für Familie, Senioren, Frauen und Jugend, Renate Schmidt, hatte 2004 gemeinsam mit der Bundesministerin für Gesundheit und Soziale Sicherung, Ulla Schmidt, den Runden Tisch Pflege ins Leben gerufen. Eine Arbeitsgruppe des Runden Tischs befasst sich speziell mit der Aufgabe, Entbürokratisierungspotenziale in der Altenpflege aufzudecken und auf breiter Basis Lösungsvorschläge zu ermitteln. Daneben wurde ein Kompetenzteam mit der Erar-

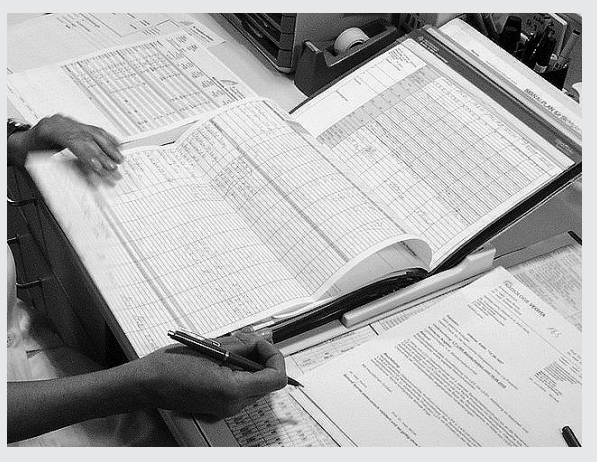

konnte auch eine Unterdokumentation in Bezug auf pflegerische Kernthemen wie Essen und Trinken oder Mobilität wahrgenommen werden. Die Studie gibt keinen Hinweis darauf, dass Über- und Unterdokumentation des Pflegeprozesses durch gesetzliche Vorschriften oder durch Vorgaben der Prüfinstanzen verursacht werden.

Insgesamt kommt die Untersuchung zu dem Ergebnis, dass eine Zentrierung der Pflegedokumentation auf die für eine beitung einer Studie zu Entbürokratisierungspotenzialen in der stationären Altenpflege beauftragt.

Ein erstes Zwischenergebnis liegt seit 15. Juni 2005 zum Thema "Pflegedokumentation « vor. Untersuchungsgegenstand war neben den Pflegeplanungs- und Dokumentationssystemen von 29 Einrichtungen insgesamt 276 einzelne Pflegedokumentationen.

Die Studie konstatiert einem Großteil der analysierten Pflegedokumentationen einen "unangemessenen Umfang und eine mangelnde Handlungsorientierung « der Pflegeplanung. Festzustellen sei eine "Überdokumentation « von Daten, die für die Pflege nicht benötigt und nicht genutzt werden. Anderseits fachgerechte und individuelle Pflege erforderlichen Inhalte nötig ist. Dazu bedarf es einerseits einer Reduzierung des Umfangs der Dokumentation und andererseits einer Vervollständigung der Inhalte. Zur Verbesserung der Situation empfehlen die Autoren daher die Erarbeitung von Handlungsleitlinien, die den jeweils Verantwortlichen Anhaltspunkte für Auswahl und Gestaltung des Pflegedokumentationssystems und Dokumentationsregeln an die Hand geben.

Quelle: http://www.pflegewiki.de/wiki/Studie_des_Runden_ Tisch_Pflege zu_Entb\%C3\%BCrokratisierungspotenzialen in der_station\% $\%$ C $\%$ A4ren_Altenpflege\#Pflegedokumentation 


\section{Auswahl der gesetzlichen Regelung}
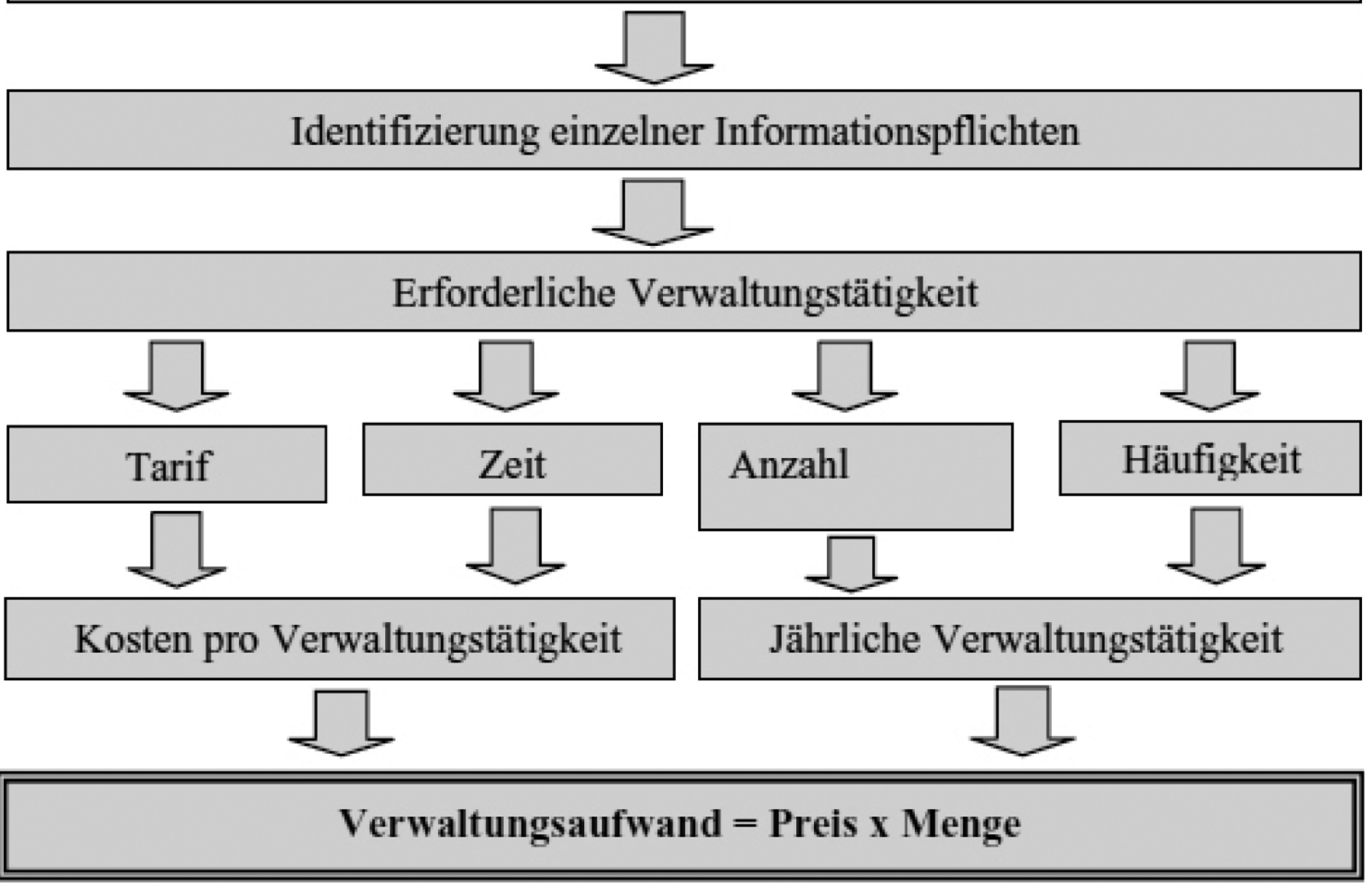

Abb. 1 Anwendung der Berechnungsformel

Quelle: Statistisches Bundesamt

hoben. Die genannten Effekte spiegeln sich wider. Der Nachbereitungsaufwand kann sowohl Ausdruck für den Qualitätsstand als auch Ausdruck von individuellen Auslegungen der Aufsichtspersonen sein.

\section{Wie das Standard-Kosten-Modell funktioniert}

Im Standard-Kosten-Modell wird der Zeitaufwand ermittelt, in dem Betroffene befragt werden, wie lange sie für die Ausführung einzelner Arbeitsschritte benötigen, beispielsweise für das Beschaffen der notwendigen Formulare, das Durchführen von Berechnungen, das Aufsetzen von Schreiben. Diese Arbeitsschritte werden »Standardaktivitäten « genannt und geben dem Modell seinen Namen.

Bei den Unternehmen wurden die Kosten des für die Erfüllung der Pflichten eingesetzten Personals und für weitere Anschaffungen in Euro ermittelt. Für die Bürgerinnen und Bürger wird der Zeitaufwand in Stunden ausgewiesen. Die so erfassten Zeiten und Kosten werden mit der Anzahl der Betroffenen in einem Jahr multipliziert. Dadurch wird der bürokratische Aufwand, den eine Informationspflicht in Deutschland verursacht, objektiv aufgezeigt. Die Ergebnisse machen die Größenordnung und Verteilung der bürokratischen Belastungen für die Öffentlichkeit deutlich.

Die Berechnung des Verwaltungsaufwands ergibt sich aus Preis und Menge (vgl. Abb. 1). Während die MDK-Kosten bei den gleichen Einrichtungen Schwankungsbreiten von 20 bis 30 Prozent aufweisen, zeigen sich bei den identischen Einrichtungen bei den Kosten der Heimbegehung Schwankungen von bis zu 500 Prozent. Ein Einzelfall? - Wir glauben nicht.

Die Bundesregierung hat bis Ende 2009 die Bürokratiebelastung der Wirtschaft aus Informationspflichten von 47,6 Milliarden Euro pro Jahr bereits um rund 6,6 Milliarden Euro durch in Kraft getretene Maßnahmen abgebaut. Das Zwischenziel auf dem Weg zur angestrebten Netto-Entlastung der Wirtschaft um 25 Prozent bis Ende 2011 im Vergleich zu 2006 ist damit erreicht.

Die Diskussion um den Grad der unternehmerischer Gestaltungsfreiheit und die zur Qualität notwendigen Prüfungen kann aus unserer Sicht nicht pauschal beantwortet werden, da sie vom » Reifegrad « des Unternehmens - und damit auch vom Management - abhängig ist. Es zeigt sich, dass die Integration der Anforderungen in $\mathrm{Ma}$ - nagementsystemen, die dann aber entsprechend im Unternehmen gelebt werden müssen, erfolgreich ist. Qualitäts- und Risikomanagement seien hier als zwei Managementsysteme genannt, die, wenn sie ernsthaft in Unternehmen gelebt werden, hilfreich sind.

Doch hier ist noch entsprechender Entwicklungsbedarf. Qualitätsmanagement gestaltet sich oftmals als Aufbau von Bürokratie im eigenen Unternehmen. Gerade in Deutschland findet sich hier eine Legitimierung von bürokratischem Handeln. Fehlentwicklungen sind dringend zu vermeiden und zurück zu entwickeln. Denn Bürokratie wird sowohl von außen als auch in den Unternehmen selbst verursacht. Gerade Qualitätsmanagementsysteme bilden hier manchmal einen eigenen Dschungel und helfen nicht, sondern verkomplizieren. Qualitätsmanagement-Diät ist angesagt.

Wenn wir über die Entbürokratisierung nachdenken, müssen wir folgende Potentiale betrachten:

überflüssige und änderungsbedürftige Vorschriften auf der Ebene Bund, Länder und Kommunen

- Offenlegung von Abstimmungsproblemen leistungs- und aufsichtsrechtlicher Vorschriften 

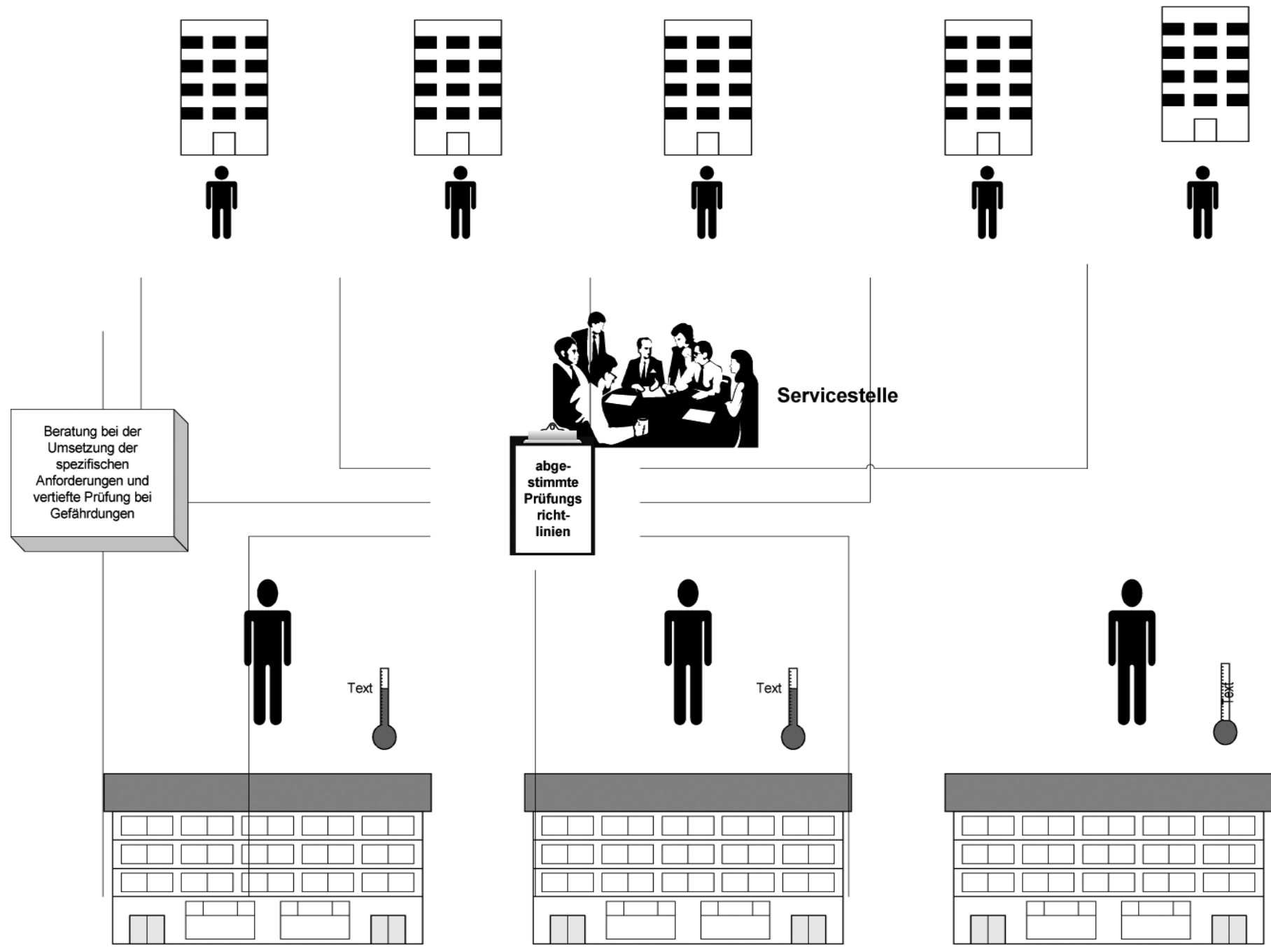

Abb. 2: Schaubild Servicecenter

- Aufdecken innerorganisatorischer Entbürokratisierungspotenziale

- spezifische Betrachtung des Pflegeplanungs- und Dokumentationsaufwandes

Doch auch bei der Pflegedokumentation sind Fehlentwicklungen zu verzeichnen. Aus den Prüfberichten geht hervor, dass auf der Inhaltsebene der Dokumentation oftmals Romane oder aussagelose Mitteilungen ohne Prioritätensetzungen zu finden sind. Auf der Strukturebene haben wir es mit einer Vielfalt von Formularen und auseinandergezogenen Unterlagen zu tun, die für den Mitarbeiter erhöhten Aufwand bedeuten. Die Dokumentation wird zum Teil losgelöst von direkter Pflege gelehrt. Doch es gibt auch erste Ansätze gemeinsamer Kooperation unterschiedlicher Behörden. So wurde im sogenannten »Kasseler Modell « die Integration der unterschiedlichen "behördlichen " Anforderungen in das Qualitätsmanagement von Einrichtungen integriert. Ein gemeinsamer und erfolgreicher Ansatz. Auch das Projekt KoBra ist ein gelungenes Beispiel für Entbürokratisierung und Selbstverantwortung.

\section{Beispiel KoBra - Kooperation Breitenumsetzung von Arbeitsschutz in der Pflege}

Im Projekt »KoBra - Kooperation Breitenumsetzung von Arbeitsschutz in der Pflege « soll der Arbeitsschutz in den Betrieben der stationären Altenhilfe landesweit verbessert und dabei innovativ zusammengearbeitet werden. Derzeit besteht in 30 bis 50 Prozent der Pflegeeinrichtungen in Baden-Württemberg massiver Optimierungsbedarf. Oft werden nicht die gesetzlichen Mindestvorschriften erreicht.

Ein Online-Tool - der »RisikoQuickCheck « (RQC - www.kobrabw.de) gibt den Einrichtungen anhand einer verdichteten Auswahl von Leitfragen die Möglichkeit, sich selbst zu prüfen und anhand einer Toolbox die notwendigen Schritte sofort einzuleiten. Hierdurch wird Rechtssicherheit erreicht und das Lernen gefördert. In nur 30 Minuten ist der Selbsttest durchgeführt und außerdem gibt es eine Rückmeldung, wo die eigene Einrichtung im Vergleich zur Branche steht.
Die freiwillige Beteiligung von fast der Hälfte der Einrichtungen spricht für einen Erfolg des Instrumentes. Mit dem gemeinsamen Projekt sollten Alternativen gefunden werden, die mit alternativen Überwachungsmöglichkeiten eine Breitenwirkung erreichen und gleichzeitig die Eigenverantwortung der Betriebe stärken. Ein weiteres Ziel des Projektes besteht darin, dass sich die Akteure auf ein gemeinsames Instrumentarium und damit zugleich auf gemeinsame Mindeststandards einigen.

\section{Vision: Servicecenter als Aufsichtsorgan}

Die Idee des Servicecenters ist angelehnt an die auf kommunaler Ebene geschaffenen Bürgerbüros, in der der Einwohner eine Breite von Leistungen durch einen behördlichen Mitarbeiter bekommen kann (vgl. Abb. 2). Dem Ansatz der Kundenorientierung folgend, in dem die Prämisse »One $\mathrm{Fa}$ ce to the customer « im Vordergrund steht, könnte dieser Gedanke auf die unterschiedlichen Aufsichtsinstitutionen übertragen 


\section{Die wissenschaftliche Fundierung von Bürokratie}

geht auf die Arbeiten von Max Weber (1864-1920, Foto) zurück, der »Bürokratie« als soziologischen Grundbegriff prägte. Wesentliche Merkmale von Bürokratien sind demnach:

(1) Ein geordnetes System von Regeln auf der Basis einer Satzung,

(2) eine hierarchisch gegliederte, unpersönliche Ordnung,

(3) die Abgrenzung von Kompetenzen und Zuordnung von Funktionen, Verantwortlichkeiten und Befugnissen,

(4) die Auslese der Funktionsträger nach formalen Qualifikationen,

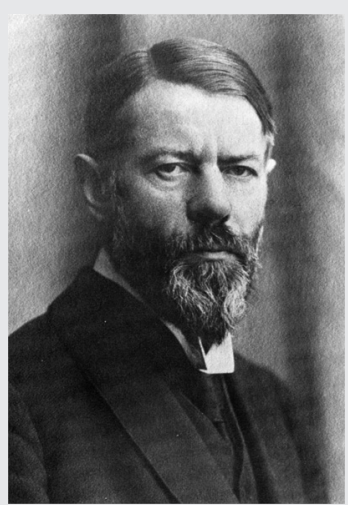

(5) das schriftliche Erfassen und Dokumentieren aller Vorgänge sowie

(6) die Konzentration von Herrschaft durch Wissen.

Als Vorteil einer bürokratischen Organisation wird allgemein vor allem ihre Objektivität, Stetigkeit, Berechenbarkeit, Planbarkeit und Zuverlässigkeit angesehen. Umgekehrt besteht dagegen die Gefahr, dass Bürokratie zum Selbstzweck wird, indem sie ihre interne Organisation gegenüber den eigentlichen Zielen in den Vordergrund stellt (Bürokratismus).

Detlef Friedrich und durch die Einrichtung einer Servicestelle entgegnet werden.

Die Servicestelle bündelt die unterschiedlichen behördlichen Anforderungen und stimmt diese zwischen den verschiedenen zuständigen Aufsichtsinstitutionen ab. Die jeweilige Einrichtung erhält einen Ansprechpartner, der für alle Fragen zuständig ist. Die hinter der Servicestelle stehenden Institutionen konzentrieren sich in ihrem Wirken auf die Beratung der Einrichtungen hinsichtlich der Umsetzung der Anforderungen im Sinne der Qualitäts- und Systemkontrolle und stehen für Vertiefungsprüfungen zur Verfügung.

Die Servicestelle ist damit das zuständige "Aufsichtsorgan«, welches anhand ent- sprechender Risikokataloge die Gefährdungslage des Betriebes im Sinne der Gefahrenabwehr überprüft.

\section{Fazit}

Wir brauchen neue Ideen und Entwicklungen. Und das sowohl bei den Aufsichtsinstitutionen als auch intern in den Unternehmen. Konzentration auf den Kernprozess - das muss wieder geschaffen werden. Modellprojekte zeigen, welche Erfolge erzielt werden können und wie lange der Weg zu einer dialogorientierten Zusammenarbeit zwischen den Institutionen dauert. Hier treffen Kulturen aufeinander, die erst einmal in ein einheitliches Verständnis gebracht werden müssen. Ein gemeinsames Kooperationsverständnis mit den Einrichtungen muss entwickelt werden.

Bei den Einrichtungen, die Anlass zu Kritik geben, ist es in der Regel eine Frage des Managements, und dies wird heute durch jede einzelne prüfende Institution festgestellt. Hier hilft ein abgestimmtes Vorgehen, weil nur dann die Verbraucherund Mitarbeiterschutzinteressen gewahrt werden und nur so eine einheitliche Entwicklung zu einer entbürokratisierten, qualitätsgesicherten Pflege erreicht werden kann. Ziel muss es werden, die Ergebniskriterien so valide zu beschreiben, dass die Struktur- und Prozessqualität in unternehmerischer Freiheit gelebt werden kann.

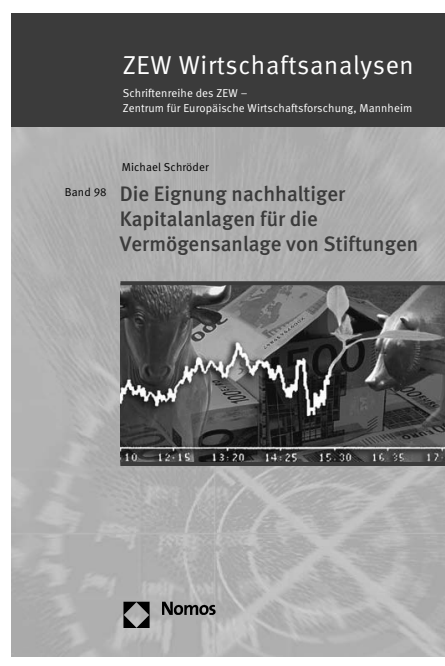

\section{Die Eignung nachhaltiger Kapitalanlagen für die Vermögensanlage von Stiftungen} Von PD Dr. Michael Schröder 2010, 262 S., brosch., 59,- $€$, ISBN 978-3-8329-5662-2 (ZEW Wirtschaftsanalysen - Schriftenreihe des ZEW, Bd. 98)

Im Zentrum der Studie stehen die Untersuchung von Strategien der Vermögensanlage und ihre Bewertung aus der Perspektive gemeinnütziger Stiftungen. Von besonderer Bedeutung sind dabei nachhaltige Kapitalanlagen, die spezielle ethische, soziale und ökologische Auswahlkriterien anwenden. Sie werden einer umfassenden betriebs- und finanzwirtschaftlichen Analyse unterzogen. 